Acta vet. scand. $1969,10,295-297$.

Brief Communication

\title{
MYCOPLASMA SUIPNEUMONIAE ISOLATED IN DENMARK
}

Though several agents may be involved in the etiology of enzootic pneumonia of pigs, recent investigations seem to indicate that the great majority of cases are caused by a Mycoplasma species - called M. suipneumoniae in Britain (Goodwin et al. 1965) and M. hyopneumoniae in the U.S.A. (Maré \& Switzer 1965 ).

In Denmark catarrhal lung lesions typical of enzootic pneumonia are frequently found in pigs at meat inspection, and investigations have for some time been carried out in order to identify the mycoplasmas present in lungs of Danish pigs.

From the lungs of one pig an agent has now been recovered which fits in well with the characteristics of M. suipneumoniae. The isolation of this agent was accomplished in a new type of medium, which has proved capable of supporting growth of $M$. suipneumoniae. The composition of this medium-FF is as follows:

Hank's balanced salt solution, $400 \mathrm{ml}$; double-distilled water, $715 \mathrm{ml}$; brain heart infusion broth (Difco), $8.5 \mathrm{~g}$; tryptic digest broth (Difco), $6.4 \mathrm{~g}$; lactalbumin hydrolysate (Difco), $2.0 \mathrm{~g}$; yeast extract (Difco), $2.0 \mathrm{~g}$. This mixture is autoclaved, and $35 \mathrm{ml}$ of a freshly prepared extract of Fleischmann's pure dry yeast type 2040, $25 \mathrm{ml} 10 \%$ glucose, 300.000 i.u. of penicillin, and $2.5 \mathrm{ml} 5 \%$ thalliumacetate added. To this basal medium $25 \%$ sterile horse serum is added. Per 100 $\mathrm{ml}$ of final medium $0.4 \mathrm{ml}$ of a $7.5 \% \mathrm{NaHCO}_{3}$ solution is added. Phenol red is used as $\mathrm{pH}$ indicator. Solid medium is prepared with special agar-Noble (Difco) in a $0.9 \%$ concentration. The agar is autoclaved in a $15 \%$ solution (double-distilled water) and added to the medium at $50^{\circ} \mathrm{C}$.

For growth inhibition tests, discs were completely soaked with rabbit hyper-immune serum against $M$. suipneumoniae ${ }^{1}$ ), dried at $37^{\circ} \mathrm{C}$, and stored at $+4^{\circ} \mathrm{C}$ until use.

Metabolic inhibition tests with rabbit hyper-immune serum against $M$. suipneumoniae ${ }^{1}$ ) were read when a $\mathrm{pH}$ decrease of 0.5 had occurred in the control tubes.

1) Type NCTC 10110 obtained from Dr. R. H. Leach, Mycoplasma Reference Laboratory, Colindale, London. 
In July 1969 five lungs of pigs originating from four unknown herds were collected at a Danish slaughter-house. All of these lungs showed lesions typical of catarrhal pneumonia.

Pneumonic lung tissue was carefully minced and suspended in fluid medium in dilutions up to $10^{-7}$. After three passages in fluid medium subcultures on solid medium were made.

In the tubes inoculated with material from four of the lungs a rapid decrease of $\mathrm{pH}$ was noted, and subcultures on solid medium showed growth of large mycoplasma colonies of the "fried-egg" type (Fig. 1) which were identified as Mycoplasma

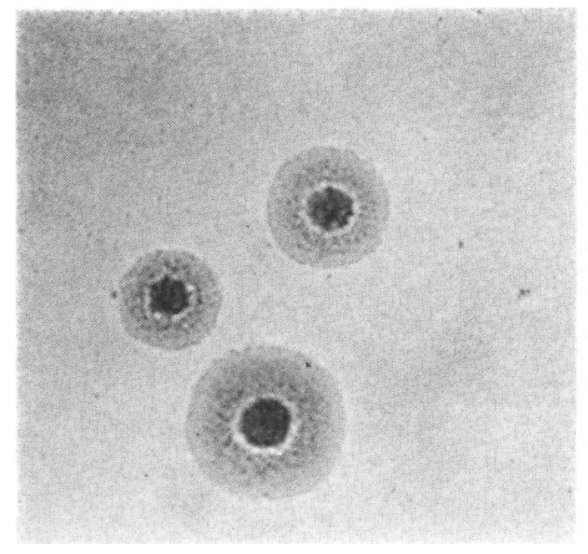

Fig u r e 1. Mycoplasma hyorhinis isolated from the lung of a Danish pig with catarrhal pneumonia. After 2 days of incubation on solid medium-FF. $\times 32$.

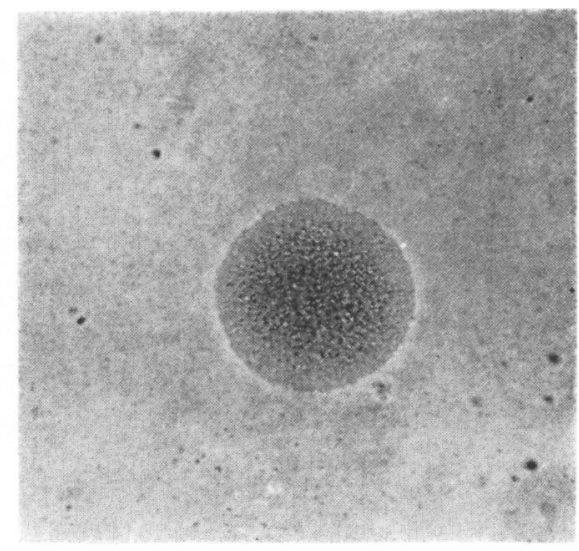

F i g u r e 2. Mycoplasma suipneumoniae isolated from the lung of a Danish pig with catarrhal pneumonia. After 6 days of incubation on solid medium-FF. $\times 32$.

hyorhinis types. The endpoint titers obtained for these four lungs were $10^{-3}, 10^{-4}, 10^{-5}$ and $10^{-7}$.

In the tubes inoculated with material from the fifth lung no growth of M. hyorhinis occurred. However, after about one week a slowly progressing decrease of $\mathrm{pH}$ was noticed. On subcultures on solid medium, colonies morphologically resembling $M$. suipneumoniae were visible after 4 days. After one week the size of the colonies ranged up to $200 \mu$ and no central nipple was visible (Fig. 2). Growth inhibition test against M. suipneumoniae antiserum showed a $3 \mathrm{~mm}$ broad zone of inhibition, and in the metabolic inhibition test with the same antiserum lack of $\mathrm{pH}$ shift was recorded up to a dilution of $1 / 128$. In the primary cul- 
tures from the lung material an endpoint titer of $10^{-3}$ was obtained.

In August 1969 six more lungs were collected, all of which showed lesions of catarrhal pneumonia. From two of these lungs, strains of mycoplasma were isolated which showed the same cultural characteristics as the one described above. Serological identification has confirmed the identity to M. suipneumoniae.

The pathogenicity of Danish isolates of $M$. suipneumoniae has not yet been ascertained. If, however, their pathogenicity for pigs is confirmed, it is reasonable to assume that this mycoplasma species is the main cause of catarrhal pneumonias in Danish pigs.

The medium-FF described in this article is a medium of a new composition. It has been confirmed that it is capable of supporting growth of M. suipneumoniae, but it is not yet known with certainty whether it is quite as well suited for primary isolation of this mycoplasma as the medium described by Goodwin et al. It is easier to prepare, however, and it has been established that its capacity of supporting growth varies very little from one batch to the other. For the isolation of other mycoplasmas from pigs, especially M. hyorhinis, it has turned out to be excellent.

Niels F. Friis

State Veterinary Serum Laboratory

Copenhagen, Denmark.

\section{REFERENCES}

Goodwin, R. F. W., A. P. Pomeroy \& P. Whittlestone: Production of enzootic pneumonia in pigs with a mycoplasma. Vet. Rec. 1965, 77, $1247-1249$.

Maré, C. J. \& W. P. Switzer: New species: Mycoplasma hyopneumoniae. A causative agent of virus pig pneumoniae. Vet. Med. $1965,60,841-846$.

(Received August 25, 1969). 


